\title{
Review
}

\section{The diagnosis and management of pre-invasive breast disease Pathological diagnosis - problems with existing classifications} Marc J Van de Vijver and Hans Peterse

\author{
Netherlands Cancer Institute, Amsterdam, The Netherlands \\ Corresponding author: Marc Van de Vijver (e-mail: m.vd.vijver@nki.nl) \\ Published: 29 July 2003 \\ Breast Cancer Res 2003, 5:269-275 (DOI 10.1186/bcr629) \\ (C) 2003 BioMed Central Ltd (Print ISSN 1465-5411; Online ISSN 1465-542X)
}

\begin{abstract}
In this review, we comment on the reasons for disagreement in the concepts, diagnosis and classifications of pre-invasive intraductal proliferations. In view of these disagreements, our proposal is to distinguish epithelial hyperplasia, lobular carcinoma in situ and ductal carcinoma in situ, and to abandon the use of poorly reproducible categories, such as atypical ductal hyperplasia or ductal intraepithelial neoplasia, followed by a number to indicate the degree of proliferation and atypia, as these are not practical for clinical decision making, nor for studies aimed at improving the understanding of breast cancer development. If there is doubt about the classification of an intraductal proliferation, a differential diagnosis and the reason for and degree of uncertainty should be given, rather than categorizing a proliferation as atypical.
\end{abstract}

Keywords: atypical ductal hyperplasia (ADH), breast, ductal carcinoma in situ (DCIS), epithelial hyperplasia, review

\section{Introduction}

The traditional models of breast cancer development are based on morphological studies and suggest the transition from a normal epithelial cell via hyperplasia and atypical hyperplasia (Fig. 1a) to ductal carcinoma in situ (DCIS). These studies were supported by analogy with mouse mammary tumour models and by epidemiological studies, which showed that the risk for breast cancer increased with the rate of proliferation and atypia in breast biopsies. Although proliferation per se is a probable risk factor for breast cancer, in our view the only intraductal proliferation that can be considered as an obligate precursor to every invasive breast cancer is carcinoma in situ. This implies that every invasive carcinoma initially develops as a carcinoma in situ (but this does not imply that every carcinoma in situ will progress to invasive cancer). Within the category of carcinoma in situ are lobular carcinoma in situ (LCIS) and DCIS. It is well established that DCIS can be further subdivided based on cytonuclear characteristics, growth pattern and absence or presence of necrosis, and that also specific genetic change indicates that there are different pathways for development of these in situ carcinomas.

There is, however, no direct evidence that epithelial hyperplasia and atypical hyperplasia are precursors to carcinoma in situ or invasive carcinoma, neither from epidemiological, nor from histopathological or molecular biological studies. Therefore, the proposal of the terms mammary intraepithelial neoplasia [1] and ductal intraepithelial hyperplasia [2], embodying the concept of continuous progression from epithelial hyperplasia to carcinoma in situ, should not be adopted (Fig. 1b). In addition, there is significant interobserver variability in diagnosing atypical ductal hyperplasia (ADH) [3], which is not resolved by giving these lesions a different name.

Lastly, it is very probable that the nature of the rare lesion originally classified as $\mathrm{ADH}$ has changed: in the premammography era this lesion was detected by chance in otherwise benign biopsies, whereas nowadays this lesion is most commonly diagnosed in radioguided biopsies

$\mathrm{ADH}=$ atypical ductal hyperplasia; $\mathrm{AL}=$ atypical lobules; $\mathrm{ALH}=$ atypical lobular hyperplasia; $\mathrm{DCIS}=$ ductal carcinoma in situ; FNA = fine needle aspiration; LCIS $=$ lobular carcinoma in situ. 
Figure 1

(a)

\section{possible general model for the multistep development of breast cancer?}

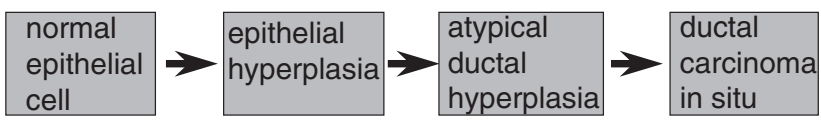

(b)

possible general model for the multistep development of breast

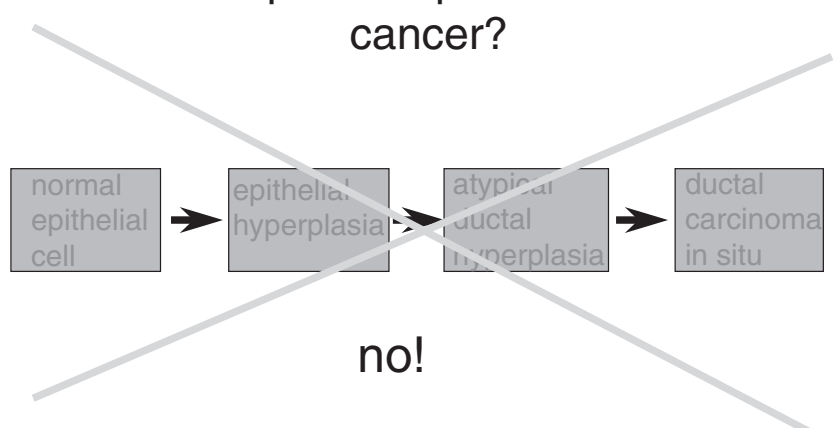

The multistep development of invasive breast cancer. (a) This model is often used to explain how breast cancer develops. The way in which the model is drawn suggests that epithelial hyperplasia is a direct precursor to atypical ductal hyperplasia (ADH) and ductal carcinoma in situ (DCIS). (b) There is no proof that epithelial hyperplasia is a direct precursor of $\mathrm{ADH}$ and DCIS, and that there is a continuum of alterations leading from epithelial hyperplasia to DCIS.

taken because of microcalcifications, or from the lesions detected by ductal lavage.

The following text sets out arguments to abandon some of the categories used or suggested to classify intraductal proliferations, especially $\mathrm{ADH}$, the so-called clinging type of $\mathrm{ADH}$, and atypical lobular hyperplasia (ALH). We will also comment on the diagnostic problems and therapeutic consequences of intraductal proliferations in core biopsies, and on the claimed role of ductal lavage in the assessment of breast cancer risk.

\section{Atypical ductal hyperplasia}

$\mathrm{ADH}$ in the presently used nomenclature is viewed as a risk factor for and not as a direct precursor of invasive breast cancer [4]. However, the first studies, at the beginning of the 20th century, leading to the concept of ADH were performed to find precancerous lesions in the breast. The method mainly used for such studies was to examine breast tissue surrounding invasive breast carcinomas. intraductal proliferations and attempts were made to categorize these; the term 'atypical hyperplasia' or 'hyperplasia with atypia' was introduced and was used mainly to indicate the presence of proliferations of epithelial cells with cytonuclear atypia. Wellings et al. have reviewed the older literature on this subject and build on it for their own studies on possible precancerous lesions [5]. Wellings et al. write that the rationale for their studies was based on their prior experience with rodent models, most notably the epithelial proliferations seen in mice with mouse mammary tumour virus induced lesions of the breast. These investigators also started to use a subgross sampling technique of breasts with cancer and breasts without malignancy. They found 'atypical lobules' (AL) more commonly in cancerous breasts compared to noncancerous breasts. They summarize the characteristics of $\mathrm{AL}$ as follows: 'a) It was more common in cancerous breasts or in those contralateral to cancer than in breasts not so identified; b) it had lobular morphology and was a terminal structure on the mammary tree; c) it tended to persist after the menopause, whereas normal lobules usually atrophied; d) it showed variable degrees of anaplasia forming an arbitrary continuum from normal lobules to ductal carcinoma in situ; and e) as AL progressed to DCIS, the unfolded lobule resembled a duct which gave the false impression that DCIS was a ductal lesion. The morphologic evidence supported the hypothesis that the lesions herein called AL were derived from TDLU [terminal ductal lobular unit] and were precancerous.' [5]

Another important area of research, pioneered by Page and co-workers, is the review of biopsies of benign breast disease from women with long-term follow-up [4]. Using a cohort-based approach and, more commonly, a casecontrol approach, the histopathological findings in biopsies from women who developed breast cancer were compared to those from women who remained free of disease. In this way, various lesions have been associated with an increased risk of developing breast cancer, including radial scars [6], fibroadenomas [7], and various forms of epithelial hyperplasia, most notably the lesion they categorized as $A D H$ [8]. The increased risk associated with these lesions was found for both breasts (and not only for the biopsy site), leading to the postulate that these lesions were 'non-obligate precursors' of breast cancer. The attempts to define categories of intraductal proliferations thus largely stems from the search for precancerous lesions, and the search for lesions associated with increased risk. The application of the terminology emerging from this work in diagnostic breast pathology has caused problems, most notably in producing appropriate definitions and applying these with acceptable interobserver variability.

It is a fact of life that some of the intraductal proliferations can pose diagnostic difficulties. The most frequently 
encountered differential diagnostic problem is whether a lesion represents epithelial hyperplasia or carcinoma in situ. This has led to the application of the findings from the work described above to define a category of intraductal proliferations that is intermediate between epithelial hyperplasia and DCIS, and which has been termed ADH.

The most commonly used definition is that $\mathrm{ADH}$ represents 'a proliferative lesion that fulfils some but not all criteria for a diagnosis of low grade, non-comedo type DCIS.' In the original series $A D H$ was described as usually unifocal and small, often less than $1 \mathrm{~mm}$ (or two spaces) in size [4].

In trying to refine these criteria, there have been many contributions in the literature, which are summarized below: 'ADH has as its upper boundary the most minimal lesions recognized as DCIS, i.e., the low grade (non-comedo) lesions, largely cribriform in pattern. The lower boundary of $\mathrm{ADH}$ is defined by examples of florid hyperplasia with focal areas of cellular uniformity and even placement of cells' [9]. Although these authors stress that their criteria for distinguishing ordinary hyperplasia and DCIS from $\mathrm{ADH}$ are not placed on a continuous scale, application of their above-mentioned description will not be helpful in separating these lesions.

As a characteristic feature of ADH 'partial involvement' of a duct has been described. This means that a duct contains a focus of a monotonous rigid cribriform or micropapillary proliferation, whereas the duct lining consists of one layer of polarized cells with more cytoplasm and vesicular nuclei. In this way also extensive monotonous proliferations with clinging, micropapillary and only focal cribriform patterns can be categorized as ADH.

As the extent of the lesion is the main determinant separating most examples of ADH from DCIS, an upper size limit was defined [10]. However, this limit varied for different situations (2-3 mm for 'standard' $A D H$, up to $4 \mathrm{~mm}$ for apocrine $A D H$ in papilloma), and was mainly applied for those lesions showing all the characteristic features of low grade DCIS.

It is questionable whether these refinements lead to improved interobserver variability in the diagnosis of $A D H$. Several studies show that the interobserver agreement on the diagnosis of $A D H$ is very poor, even when consensus about diagnostic criteria exists [3].

A very important factor that is often ignored in the recent publications on ADH is the clinical context that has led to taking a breast biopsy. If a tumour mass is present and an intraductal proliferation is noted at histological examination, the biopsy is usually not representative for the lesion. If the mass represents an invasive breast carcinoma, accompanied by a small amount of well-differentiated
DCIS, it is possible to obtain a small amount of well-differentiated DCIS in a core needle biopsy, which, according to most present classification systems, would be labelled $\mathrm{ADH}$. This is not a very practical approach: it is better to note that the biopsy is most likely not representative of the lesion and that the presence of a small amount of well-differentiated DCIS leads one to suspect that the surroundings of an invasive carcinoma have been biopsied.

Some reports also describe the presence of ADH adjacent to DCIS. This does not make any sense: the intraductal proliferation with some but maybe not all criteria for DCIS that is present adjacent to DCIS will, in most instances, be the same clonal proliferation as the dominant focus of DCIS, and should therefore be classified as such.

So, what of the remaining problematic situations with diagnosis? A biopsy may contain only a very small amount of DCIS, or a biopsy may show an intraductal proliferation that cannot be easily classified as DCIS or epithelial hyperplasia. If a very small amount of DCIS is found, our proposal is simply to make the diagnosis of DCIS and specify that the lesion is very small. When an intraductal proliferation cannot be classified with certainty, this should be indicated; for example, the differential diagnosis for intraductal epithelial proliferation would be well-differentiated DCIS or epithelial hyperplasia.

\section{Atypical lobular hyperplasia}

For ALH the situation has become relatively simple. According to most definitions, ALH is 'a small amount of LCIS'. The original follow-up studies suggested differences in risk between ALH and LCIS, and this was the main reason to distinguish these lesions. All cases of ALH also stain negative for E-cadherin, indicating that the genetic alteration that is characteristic for LCIS is also present in these very small lesions [11]. Moreover, with longer follow-up the risk of both lesions becomes more or less comparable [12]. Therefore, there is no place for the term ALH.

There has also been extensive discussion about whether the term LCIS should be maintained. As with other intraductal proliferations, an important question in these discussions was whether LCIS is a precursor lesion or a determinant of risk. Again, the early epidemiological studies suggested an increased risk of developing cancer anywhere in both breasts, consistent with the concept that LCIS is a determinant of risk. However, recently updated prospective follow-up series $[12,13]$ show occurrence of invasive cancer preferably in the ipsilateral breast at the site of biopsied LCIS, consistent with LCIS being a precursor. The ultimate risk associated with this lesion is not lower than that for well-differentiated DCIS. We therefore prefer to use the term LCIS, complemented by additional information on the extent of the lesion. 


\section{Atypical ductal hyperplasia of the clinging type; flat epithelial atypia}

In recent years, screening with mammography is being performed with increasing frequency to detect breast cancer at an early stage. Using mammography screening, microcalcifications are a relatively frequent finding, leading to guide-wired excisions or core needle biopsies from these areas of microcalcification. As a result, breast lesions leading to microcalcifications are seen by pathologists with increasing frequency.

A particular lesion detected by microcalcifications is described by Fraser et al. [14]: 'We have noted in breast biopsies performed for microcalcifications a spectrum of lesions in the terminal duct lobular unit characterised by columnar epithelial cells with prominent apical cytoplasmic snouts, intraluminal secretions, and varying degrees of nuclear atypia and architectural complexity. The appearance of some of these lesions is worrisome, but diagnostic difficulties arise because the histological features do not fulfil established criteria for the diagnosis of atypical ductal hyperplasia or ductal carcinoma in situ (DCIS). We have termed such lesions columnar alteration with prominent apical snouts and secretions (CAPSS).'

However, this lesion was by no means new; for example, a description was given in the seminal book on breast disease by Azzopardi [15]: 'There is another, more common form of clinging carcinoma in which the lesion shows no evidence of having originated as a comedo cancer and this is indeed the most difficult type to recognize. The involved structures are lined by a single or a few layers of neoplastic cells..., showing orientation towards the lumen, whilst there is no obvious necrosis.' In his description, Azzopardi points out that usually several lobules are involved; that the cytoplasm may show lumenal blebbing; and that the myoepithelial layer is usually inconspicuous (in contrast to adenosis), and that trabecular bars and bridges may be present, which are helpful in diagnosing this lesion. He even describes the granular calcifications originating from inspissated lumenal contents, which are the reason for pick-up on mammography. The microcalcifications that lead to the excision of these lesions are usually fine and granular with multinodular/ lobular arrangements, and often involve an area of several centimetres.

There is no agreement about the nature of this lesion; concepts vary from an ordinary benign change, to the first recognizable stage of well-differentiated DCIS. This lack of consensus is reflected in the different terms used in literature: atypical lobule; cystic change; atypical cystic alteration; flat epithelial hyperplasia; and the aforementioned columnar alteration with prominent apical snouts and secretions and clinging type of DCIS.
We consider this lesion as well-differentiated DCIS clinging/micropapillary type. The arguments that this is a neoplasm rather than a form of hyperplasia include: these lesions have a similar distribution to other forms of DCIS; ducts and lobules are affected in a continuous way; and when these lesions become large, they involve only one segment of the breast.

In virtually every case of well-differentiated DCIS, there are parts of the lesion showing the exact same morphology as well-differentiated DCIS clinging/micropapillary type (Fig. 2). The cytonuclear morphology of the cells with a clinging growth pattern in these cases is identical to that of the areas with a cribriform growth pattern, although differences in cell size as a result of cell orientation (cross in the cribriform centres, perpendicular in the clinging duct lining) may suggest two cell types. Clinging DCIS is often present in and around tubular carcinoma, especially in cases with multiple invasive foci, and seems to be the noninvasive precursor of that carcinoma. In our opinion, all cases of fully developed cribriform DCIS and many cases of invasive ductal carcinoma grade 1/tubular carcinoma develop via well-differentiated DCIS clinging/micropapillary type.

The clinical question is what to do when, in a diagnostic biopsy, only well-differentiated DCIS clinging/micropapillary type is detected. At present, it is not known what percentage of these lesions will progress to become more advanced lesions and how long this progression takes. In view of the lack of consensus in recognizing and typing these lesions, which were only rarely biopsied in the premammography era, there is limited clinical experience to form the basis for clinical decision making.

Eusebi et al. found that, at a mean follow-up of 17.5 years, only two out of 32 cases of pure clinging well-differentiated DCIS treated by incision biopsy alone recurred; in both cases the recurrence was a well-differentiated DCIS [16]. The European Organization for Research and Treatment of Cancer has performed a randomized clinical trial, comparing excision alone with excision followed by radiotherapy. The cases of DCIS in this study were classified histologically by central pathology review; the recurrence rate was evaluated at a median follow-up of 5.4 years [17]. No recurrences were observed in the 59 patients with well-differentiated DCIS with a clinging architecture. A low rate of invasive recurrence was also found in well-differentiated DCIS with a micropapillary growth pattern (7 out of 98 cases), whereas in well-differentiated cribriform DCIS the rate was similar to that in poorly differentiated DCIS. This suggests that these architectural patterns reflect a spectrum of development from relatively innocent clinging lesions to those with a fully developed cribriform DCIS; lesions with a micropapillary growth pattern can be considered an intermediate in this pathway. 


\section{Figure 2}
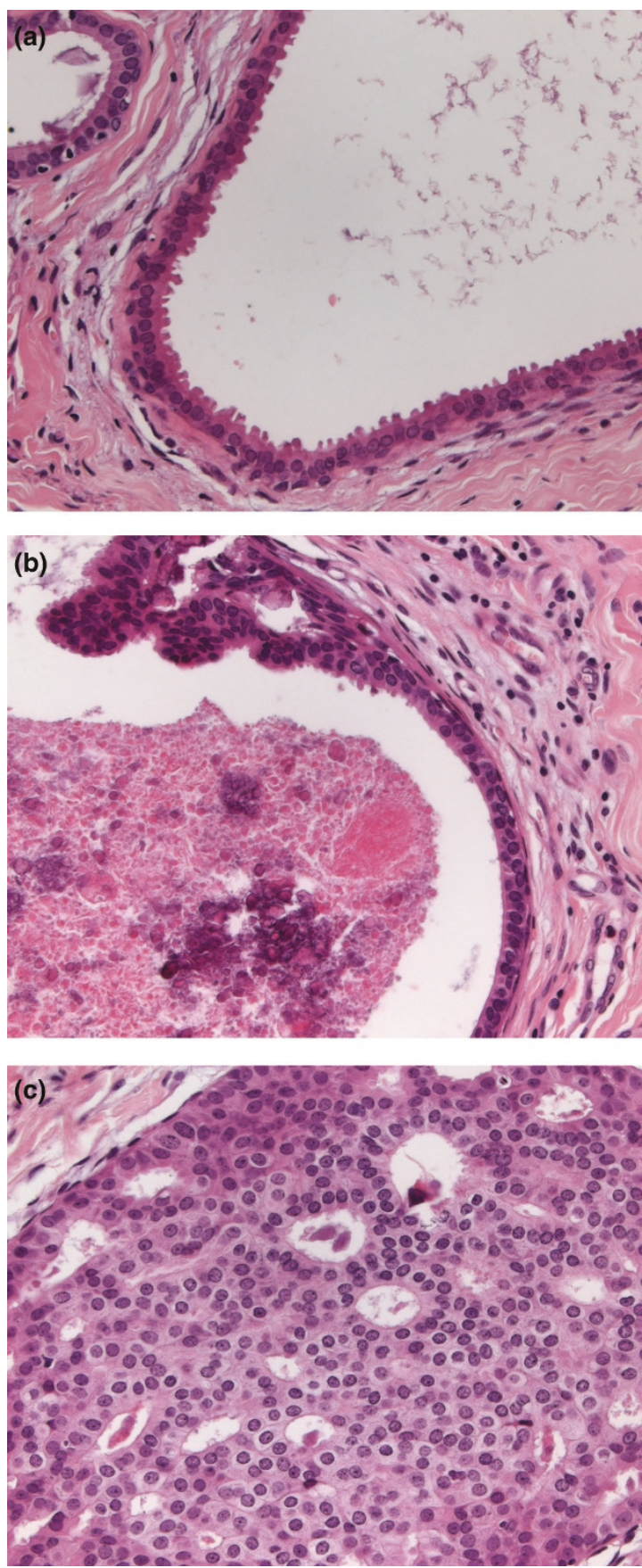

Three histological sections from one lesion. In our opinion, this lesion should be diagnosed as well-differentiated ductal carcinoma in situ (DCIS). These lesions are usually large (several centimetres in diameter). Well-differentiated DCIS often shows various growth patterns: clinging; micropapillary; cribriform. Every well developed cribriform DCIS [as shown in (c)] also contains areas with a clinging growth pattern (a). (a) Often diagnosed as columnar alteration with prominent apical snouts and secretions. (b) Often diagnosed as atypical ductal hyperplasia. These lesions are all in the spectrum of neoplasms characterized by a clonal proliferation of epithelial cells with monotonous round to oval nuclei, and for this reason they can best be classified as well-differentiated DCIS.
Clearly, more data are required to optimize clinical management for these patients. At this moment, follow-up of these patients with annual mammography seems a reasonable option. Excision of these lesions with clear margins is often difficult to achieve because the lesions are often extensive. Breast ablation appears to be overtreatment for these patients (a situation very similar to LCIS; actually, these lesions also co-exist quite frequently with LCIS).

Concerning the preferable terminology for these lesions, flat epithelial atypia has been proposed. This term avoids the term 'cancer' in the diagnosis, thus reducing patient stress and surgical overtreatment. From a conceptual point of view, however, we would prefer the term 'well-differentiated clinging type of DCIS'. When reporting this diagnosis, its relatively innocent nature should be stressed.

\section{Risk assessment for breast cancer by ductal lavage and 'random' fine needle aspiration}

Recently, several studies reported on the assessment of risk of development of breast cancer based on cytonuclear characteristics of cells obtained by minimal or non-invasive methods. Ductal lavage was used to obtain cells from the ductal system of the breast. Cytological examination was used to identify 'atypical ductal hyperplasia', or mildly and markedly atypical cells in these specimens. Likewise, multiple fine needle aspirations (FNA), taken randomly from the breasts of asymptomatic women, have been evaluated to detect cytological changes indicative of epithelial hyperplasia with or without atypia.

In 1990, Skolnick et al. reported on FNA, randomly taken from both breasts of women with and without a family history of breast cancer [18]. They reported proliferative breast disease to be more frequent in women with a family history of breast cancer. In response, Page and Dupont commented that proliferative breast disease cannot be reliably assessed on FNA, and that $\mathrm{ADH}$ certainly cannot be identified in this way [19].

It is, of course, not to be expected that ADH can be diagnosed based on cytological specimens. Because ADH is by definition a lesion of small extent and usually unifocal and because the interobserver variability is already high for the histological diagnosis, it is clear that cytological examination to diagnose ADH will perform even worse, especially as architectural features (which cannot be evaluated at cytological evaluation) play an important role in reaching this diagnosis. Evaluation of epithelial hyperplasia in cytological material has the same drawbacks: the criterion of hyperplasia is multilayered ductal lining as a result of intralumenal cell proliferation. High cellularity in FNAs does not necessarily imply epithelial hyperplasia. 
The same limitations apply to studies of ductal lavage or nipple fluid cytology. Each breast has approximately 15 segments, all ending in a separate collecting duct in the nipple. When ductal lavage is performed, usually only two to three of the collecting ducts are sampled. The sampled collecting ducts are selected on the basis of production of some fluid applying a vacuum. The unproven hypothesis is that ductal trees that do not produce some fluid on applying a vacuum will not contain epithelial hyperplasia. In any case, it is important to realize that only a small proportion of the ductal system of the breast is sampled by ductal lavage. To provide an indication of the design and outcome of such studies, one of the few large studies on fluid from the ductal system is from Wrensch and co-workers [20]. They collected nipple aspirate fluid from two series of women; the median times of follow-up were 21 years and 9 years, respectively, and breast cancer incidences were $7.8 \%$ ( 285 cases in the 3633 women for whom breast cancer status could be determined) and 3.5\% (115 of 3271), respectively. Compared with women from whom no fluid was obtained, incidences and adjusted relative risks for women in group 1 with epithelial hyperplasia and atypical hyperplasia in aspirates were slightly elevated, with very wide confidence intervals [20].

In summary, as indicated by Khan et al. 'data regarding the impact of these techniques on the detection of significant breast disease are very scant. It is important at the outset of the assessment of this new technology that breast cancer clinicians and clinical researchers think carefully about the standards of evidence that need to be met regarding the benefits of these procedures before they are widely adopted.' [21]

\section{Discussion}

As a result of the confusion with respect to the classification of intraductal proliferations of the breast there have been several proposals to introduce new classification systems, such as mammary or ductal intraepithelial proliferation. In contrast, our proposal is to return to an older classification system, where the only diagnostic subgroups are carcinoma in situ and epithelial hyperplasia. In this brief overview, we have indicated that there is no good underlying scientific evidence for the introduction or use of other diagnostic categories, nor is there evidence that the use of other diagnostic categories resolves the problems with interobserver variability in classifying intraductal breast lesions.

The classification of intraductal breast lesions is important in surgical pathology, where treatment decisions are based on diagnostic classification; in addition, the classification system forms the basis for genetic studies aimed at elucidating the multistep development of breast cancer.

For clinical purposes, the majority of intraductal prolifera-
This article is the fifth in a review series on The diagnosis and management of pre-invasive breast disease - current challenges, future hopes, edited by Sunil R Lakhani.

Other articles in the series can be found at http://breast-cancer-research.com/articles/reviewseries.asp?series $=$ bcr_Thediagnosis

hyperplasia or carcinoma in situ (usually ductal; i.e. DCIS). For the small group of lesions where no definite judgement can be given, the problem is usually that there is doubt between well-differentiated DCIS or epithelial hyperplasia. Instead of, for example, seeking outside consultation, these lesions are now often diagnosed as $\mathrm{ADH}$, a diagnosis associated with very high interobserver variability, even among expert breast pathologists. When a differential diagnosis between well-differentiated DCIS and epithelial hyperplasia is given, this should result in combining the clinical, radiological and pathological findings in a multidisciplinary discussion, which will often resolve the problem or lead to a practical clinical approach.

A good example of the presence of a specific genetic alteration in a specific type of intraductal proliferation is the inactivation of E-cadherin in lobular neoplasia. It may well be that, in the future, genetic classification will greatly help in classifying other intraductal proliferations as well.

\section{Competing interests}

None declared.

\section{References}

1. Rosai J: Borderline epithelial lesions of the breast. Am J Surg Pathol 1991, 15(3):209-221.

2. Tavassoli FA: Ductal carcinoma in situ: introduction of the concept of ductal intraepithelial neoplasia. Mod Pathol 1998, 11:140-154.

3. Sloane JP, Amendoeira I, Apostolikas N, Bellocq JP, Bianchi S, Boecker W, Bussolati G, Coleman D, Conolly CE, Eusebi V, De Miguel C, Dervan P, Drijoningen R, Elston CW, Faverley D, Gad A, Jacquemier J, Lacerda M, Mertinez-Penuela J, Munt C, Peterse JL, Rank F, Sylvan M, Tsakraklides V, Zafrani B: Consistency achieved by 23 European pathologists from 12 countries in diagnosing breast disease and reporting prognostic features of carcinomas. European Commission Working Group on Breast Screening Pathology. Virchows Arch 1999, 434:3-10.

4. Page DL, Dupont WD, Rogers LW, Rados MS: Atypical hyperplastic lesions of the female breast. A long-term follow-up study. Cancer 1985, 55(11):2698-2708.

5. Wellings SR, Jensen HM, Marcum RG: An atlas of subgross pathology of the human breast with special reference to possible precancerous lesions. J Natl Cancer Inst 1975, 55:231273.

6. Jacobs TW, Byrne C, Colditz G, Connolly JL, Schnitt SJ: Radial scars in benign breast-biopsy specimens and the risk of breast cancer. N Engl J Med 1999, 340:430-436.

7. Dupont WD, Page DL, Parl FF, Vnencak-Jones CL, Plummers WD, Rados MS, Schuyler PA: Long-term risk of breast cancer in women with fibroadenoma. N Engl J Med 1994, 331:10-15.

8. Dupont WD, Page DL: Risk factors for breast cancer in women with proliferative breast disease. N Engl J Med 1985, 312:146151. 
9. Jensen R, Page DL: Epithelial hyperplasia. In The Breast. 3rd edn. Edited by Elston CW, Ellis IO. Edinburgh: Churchill Livingstone; 2003:75.

10. Tavassoli FA, Norris $\mathrm{HJ}$ : A comparison of the results of long term follow-up of atypical intraductal hyperplasia and intraductal hyperplasia of the breast. Cancer 1990, 65:518-529.

11. Gamallo C, Palacios J, Suarez A, Pizarro A, Navarro P, Quintanilla $\mathrm{M}$, Cano A: Correlation of E-cadherin expression with differentiation grade and histological type in breast carcinoma. Am J Pathol 1993, 142:987-993.

12. Page DL, Schuyler PA, Dupont WD, Jensen RA, Plummer WD, Jr, Simpson JF: Atypical lobular hyperplasia as a unilateral predictor of breast cancer risk: a retrospective cohort study. Lancet 2003, 361:125-129.

13. Ottesen GL, Graversen HP, Blichert-Toft M, Christensen IJ, Andersen JA: Carcinoma in situ of the female breast. 10 year follow-up results of a prospective nationwide study. Breast Cancer Res Treat 2000, 62:197-210.

14. Fraser JL, Raza S, Chorny K, Connolly JL, Schnitt SJ: Columnar alteration with prominent apical snouts and secretions: a spectrum of changes frequently present in breast biopsies performed for microcalcifications. Am J Surg Pathol 1998, 22: 1521-1527.

15. Azzopardi J: Problems in Breast Pathology. Philadelphia: WB Saunders Co Ltd.; 1979.

16. Eusebi V, Feudale E, Foschini MP, Micheli A, Conti A, Riva C, DiPalma S, Rilke F: Long-term follow-up of in situ carcinoma of the breast. Semin Diagn Pathol 1994, 11:223-235.

17. Bijker N, Peterse JL, Duchateau L, Julien JP, Fentiman IS, Duval C, DiPalma S, Simony-Lafontaine J, de Mascarel I, van de Vijver M: Risk factors for recurrence and metastasis after breastconserving therapy for ductal carcinoma-in-situ: analysis of European Organization for Research and Treatment of Cancer Trial 10853. J Clin Oncol 2001, 19:2263-2271.

18. Skolnick MH, Cannon-Albright LA, Goldgar DE, Ward JH, Marshall CJ, Schumann GB, Hogle H, McWorther WP, Wright EC, Tran TD: Inheritance of proliferative breast disease in breast cancer kindreds. Science 1990, 250:1715-1720.

19. Page DL, Dupont WD: Proliferative breast disease: diagnosis and implications. Science 1991, 253:915-916.

20. Wrensch MR, Petrakis NL, Miike R, King EB, Chew K, Neuhaus J, Lee MM, Rhys M: Breast cancer risk in women with abnormal cytology in nipple aspirates of breast fluid. J Natl Cancer Inst 2001, 93:1791-1798.

21. Khan SA, Baird C, Staradub VL, Morrow M: Ductal lavage and ductoscopy: the opportunities and the limitations. Clin Breast Cancer 2002, 3:185-191.

\section{Correspondence}

Marc Van de Vijver, Dept. of Pathology, Netherlands Cancer Institute, Plesmanlaan 121, 1066 CX Amsterdam, The Netherlands. Tel: 3120 5122750; e-mail: m.vd.vijver@nki.nl 